\title{
Aspectos epidemiológicos da fibrilação atrial
}

\author{
Epidemiological aspects of atrial fibrillation
}

\section{Fernanda Augusto Justo ${ }^{1}$, Ana Flávia Garcia Silva ${ }^{2}$}

Justo FA, Silva AFG. Aspectos epidemiológicos da fibrilação atrial / Epidemiological aspects of atrial fibrillation. Rev Med (São Paulo). 2014 jan.-mar.;93(1):1-13.

\begin{abstract}
RESUMO: Fibrilação atrial (FA), a arritmia cardíaca mais comum com relevância clínica, é estudada no presente trabalho segundo aspectos epidemiológicos. Foram revisados artigos de 1947 a abril de 2013, buscando "fibrilação atrial" e "epidemiologia", "rastreamento", "mortalidade", "morbidade", "fatores de risco", entre outros em base de dados internacional, considerando fator de impacto e língua inglesa. Os resultados foram apresentados em: Perfil Epidemiológico, com prevalência, incidência, fatores de risco e etiologia; Perfil de Morbimortalidade, com comorbidades associadas mais prevalentes e causas e taxas de óbitos e; Perfil de Hospitalização, com características e tempo de internação dos indivíduos portadores da arritmia. Assim, observou-se que: 1. O aumento da expectativa de vida vem acompanhado por maior incidência de FA, com prevalência maior em grupos de idade avançada e em homens; 2. Doenças cardiovasculares são condições associadas que agravam o quadro da arritmia e as taxas de óbito; 3 . Houve aumento nos gastos hospitalares e nas internações atribuídos à FA, em discordância com a redução no número total de internações.
\end{abstract}

DESCRITORES: Fibrilação atrial/epidemiologia; Epidemiologia; Prevalência; Morbidade; Hospitalização.

\begin{abstract}
Atrial fibrillation (AF) is the most common cardiac arrhythmia clinically relevant. In this project we studied the disease based on epidemiological characteristics. Articles from 1947 to April of 2013 were reviewed, matching "atrial fibrillation" and "epidemiology", "screening", "mortality", "morbidity", "risk factors" among others in an international database, taking into consideration impact factor and English language. Results are presented as following: Epidemiological Profile, that analyses prevalence and incidence of AF regarding to age, gender, risk factors and etiology; Morbimortality Profile, that investigates the most commonly associated diseases and their multivariate mortality rates; and Hospitalization Profile, that shows hospitalized AF patients' characteristics and length of stay. Thus, it has been shown that: 1 . The increase in life expectancy is accompanied by an increase in AF incidence. The prevalence is significantly higher in men and in older people; 2 . Cardiovascular diseases are associated conditions that worsen the AF' severity and mortality rates; 3 . There was an increase in hospital expenses and in hospitalizations assigned to $\mathrm{AF}$, in spite of a decrease in hospitalizations in general.
\end{abstract}

KEY WORDS: Atrial fibrillation/epidemiology; Epidemiology; Prevalence; Morbidity; Hospitalization.

Prêmio Monografias - COMU 2013 - XXX II Congresso Médico Universitário da FMUSP.

${ }^{1}$ Acadêmica do $3^{\circ}$ ano de graduação em medicina na Faculdade de Medicina da Universidade de São Paulo, aluna de iniciação científica no Instituto do Coração do HCFMUSP. E-mail: fernandajusto@gmail.com.

2. Acadêmica do $4^{\circ}$ ano de graduação em medicina na Faculdade de Medicina da Universidade de São Paulo; aluna de iniciação científica no Instituto do Coração do HCFMUSP.

Endereço para correspondência: Fernanda Augusto Justo. Avenida Prefeito Fábio Prado, 670 - Apt. 83 - Jardim Vila Mariana, São Paulo, SP, Brasil. C.E.P.: 04116-000. E-mail: a.fernandajusto@gmail.com 


\section{INTRODUÇÃO}

fibrilação atrial(FA) é a forma mais comum
de arritmia cardíaca com importância
clínica. Trata-se de uma arritmia supraventricular gerada por alterações eletrofisiológicas que provocam ativação de focos ectópicos nos átrios e veias pulmonares ${ }^{1,2,3}$ e formação e ativação de circuitos de reentrada atrial ${ }^{1,4}$, levando a batimentos atriais desordenados com frequências superiores a 350 batimentos por minuto.

Diversos estudos mostram que sua ocorrência está associada a determinados fatores precursores, sobretudo relacionados a doenças cardiovasculares. Além de condições predisponentes que culminam com fibrilação atrial, a própria arritmia é fator de risco para o desenvolvimento de doenças cardiovasculares, aumentando, por exemplo, em cinco vezes ${ }^{5}$ a chance de um indivíduo sofrer um acidente vascular cerebral isquêmico. Quando sintomática, a fibrilação atrial pode causar limitação de funcionalidade e redução da qualidade de vida. Nesse sentido, reconhecer a epidemiologia da fibrilação atrial apresenta-se como questão de saúde pública no que diz respeito à incapacitância provocada pela doença e aos custos gerados no sistema de saúde.

\section{METODOLOGIA}

Os descritores do presente trabalho são: fibrilação atrial, epidemiologia, prevalência, morbidade, hospitalização. A captação dos artigos revisados foi feita a partir da base de dados PubMed, mantida pelo norteamericano National Center for Biotechnology Information (NCBI). Buscando-se por revisões e ensaios clínicos, "fibrilação atrial" (45.385 resultados) e os seguintes termos foram cruzados: “epidemiologia" (7.839), "rastreamento" (14.967), "mortalidade" (6.409), "fatores de risco" (13.151), "acidente vascular cerebral" (8.528), "hospitalização" (1.955), "febre reumática" (1.060), "tratamento de reposição hormonal" (59). Os artigos foram selecionados segundo fator de impacto e língua inglesa e constam na seção de "Referências Bibliográficas", que engloba publicações de 1947 a abril de 2013.

\section{Perfil epidemiológico}

A prevalência de fibrilação atrial na população geral é de aproximadamente $0,4 \%$ na população geral ${ }^{6}$. A incidência da condição está diretamente relacionada à idade, chegando a cerca de $2 \%$ em indivíduo acima de $60 \operatorname{anos}^{6}$. O estudo de Framingham, um estudo de coorte observacional prospectivo realizado nos Estados Unidos, iniciou-se em 1948, acompanhando 5209 pessoas para analisar o desenvolvimento de doenças cardiovasculares, e continua em andamento até os dias de hoje, atualmente na terceira geração de participantes. O gráfico (Figura 1), referente à incidência acumulada de fibrilação atrial, divide os grupos etários do estudo de Framingham ${ }^{7}$ segundo as idades no início do estudo e os apresenta de forma cumulativa, evidenciando crescente incidência em indivíduos mais velhos ${ }^{8}$.

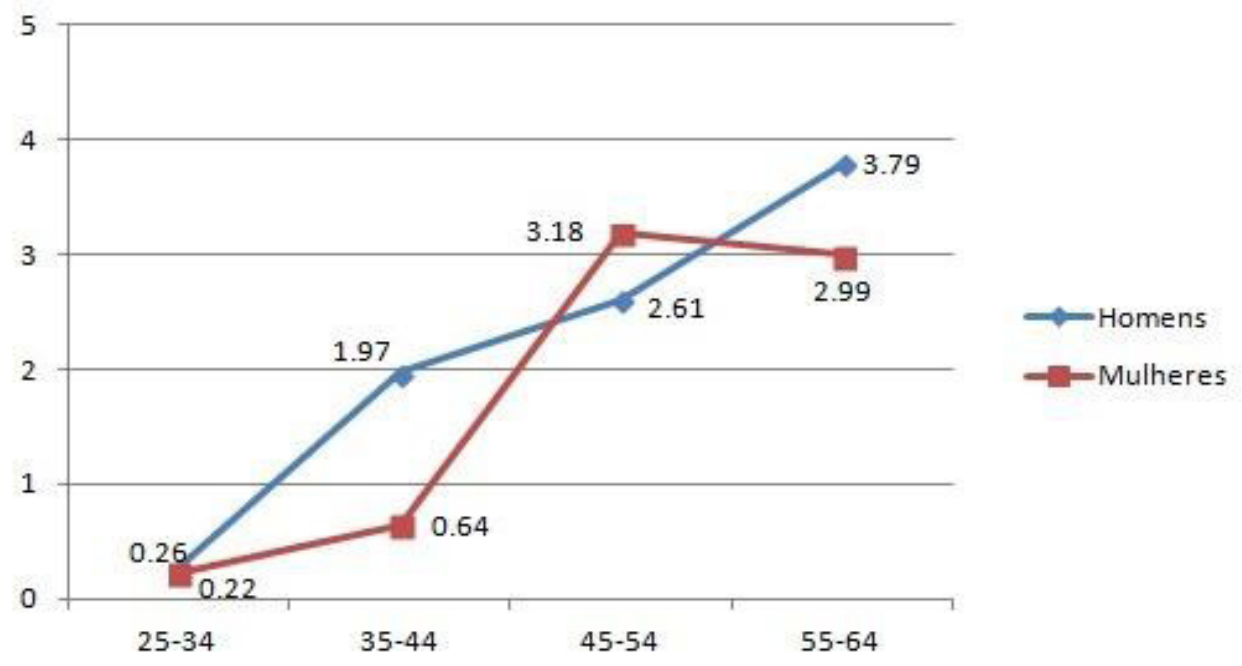

Figura 1 - Incidência (em porcentagem) acumulada de fibrilação atrial ao longo de 22 anos do estudo de Framingham segundo faixa etária no exame inicial e gêneros ${ }^{7}$

Com o envelhecimento da população, os números associados à doença devem aumentar de maneira importante, em uma razão de cerca de 2,5 vezes no caso da população americana, que em 1995 apresentava 2,3 milhões 
e em 2050 deverá apresentar 5,7 milhões de portadores de fibrilação atrial ${ }^{5}$ (Figura 2). Esse crescimento é explicado pela tendência mundial de aumento da expectativa de vida e também pela explosão populacional provocada pelo baby-boom no pós Segunda Guerra Mundial. A proporção de idosos acima de 80 anos portadores de fibrilação atrial deverá passar de 37 para 53\%, totalizando 2,95 milhões de pessoas $^{5}$. A prevalência da doença deve seguir maior em homens; entretanto, estima-se que metade dos portadores serão mulheres, reflexo da maior expectativa de vida entre elas. A Figura 3, adaptada de Thomas et al. ${ }^{10}$, mostra a distribuição da prevalência de fibrilação atrial em grupos etários sucessivos de acordo com estudos publicados até 2012. Em tais estudos, realizados na Europa, América, Ásia e Oceania, observou-se que a doença é mais prevalente em pessoas mais velhas, resultado principalmente da maior prevalência de outras condições cardiovasculares, como discutido adiante.

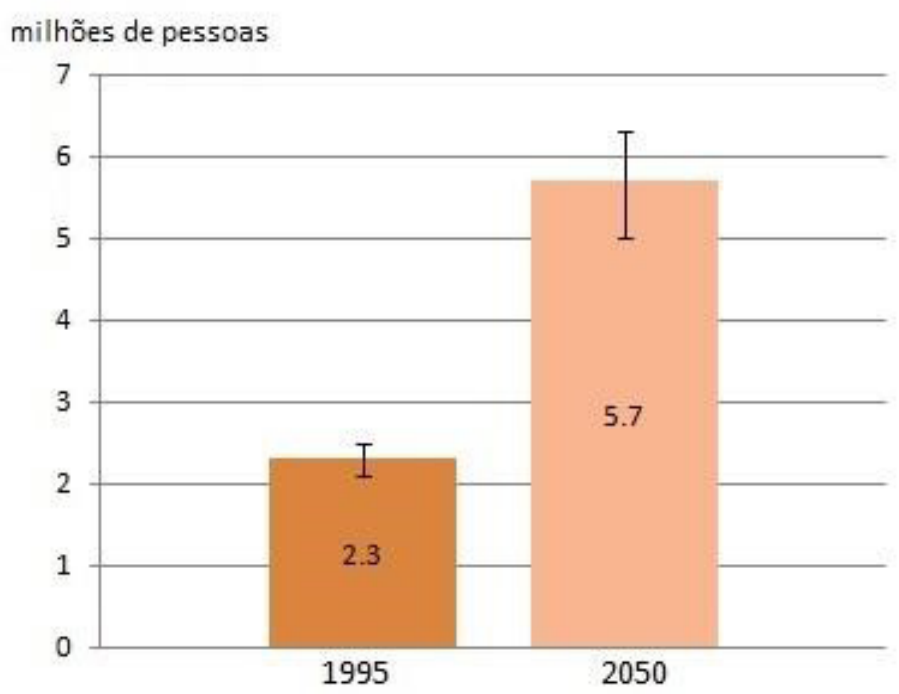

Figura 2 - Número absoluto de portadores de fibrilação atrial na população americana em 1995 e a previsão para 2050 de acordo com projeções a partir do censo norte-americano ${ }^{5}$

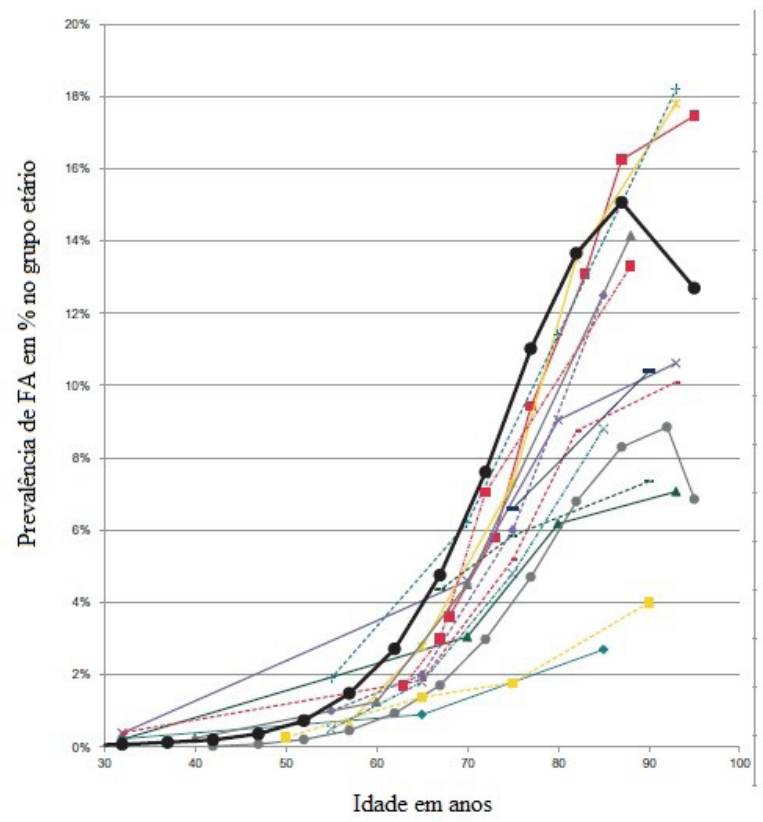

\begin{tabular}{|c|c|c|}
\hline Autor & Pais & Período \\
\hline$\rightarrow$ Ohsawa et al., 2005 & Japão & $1980-2000$ \\
\hline - Piccini et al., 2012 & EUA & 1991-2007 \\
\hline$\rightarrow$ Murphy et al., 2007 & Escócia & 2001-2002 \\
\hline * Majeed et al., 2001 & Reino Unido & 1994-1998 \\
\hline Heering et al., 2006 & Holanda & $1990-1993$ \\
\hline$\rightarrow$ Rietbrock et al., 2008 & Reino Unido & $1993-2005$ \\
\hline Miyasaka et al., 2006 & EUA & $1980-2000$ \\
\hline Go et al., 2001 & EUA & 1996-1997 \\
\hline --- Furberg et al., 1994 & EUA & n.a. \\
\hline N-... Naccarelli et al., 2009 & EUA & 2004-2005 \\
\hline$\cdots$ Jeong, 2005 & Coréia & 2000 \\
\hline$\rightarrow$ Phillips et al., 1990 & EUA & n.a. \\
\hline -Wolf et al., 1991 & EUA & $1948-1990$ \\
\hline - Lake et al., 1989 & Austrália & $1966-1983$ \\
\hline - Bonhorst et al., 2010 & Portugal & n.a. \\
\hline - Wilke et al., 2012 & Alemanha & $2006-2008$ \\
\hline
\end{tabular}

Figura 3 - Tabela adaptada de Thomas et al. ${ }^{10}$, mostra distribuição da prevalência de fibrilação atrial em grupos etários sucessivos nos estudos publicados até 2012 
A prevalência e a incidência de fibrilação atrial são maiores em homens do que em mulheres $5,7,9,10,11$ (Figura 4). De acordo com o estudo de Framingham, a incidência de fibrilação atrial por 1.000 pessoas-ano é de 3,1 em homens e 1,9 em mulheres entre 55 e 64 anos e de 38,0 em homens e 31,4 em mulheres entre 85 e 94 anos. Entretanto, há evidências de que fibrilação atrial seja mais letal em mulheres do que em homens, reduzindo, assim, a vantagem da mulher em termos de sobrevivência ${ }^{7,11}$.

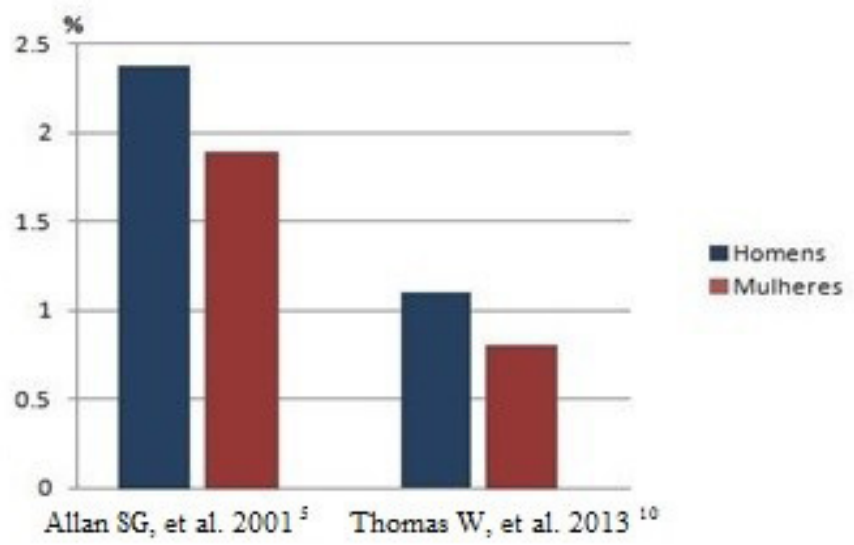

Figura 4 - O gráfico mostra a prevalência de fibrilação atrial por gênero em estudos norte-americano ${ }^{5}$ e alemão ${ }^{10}$

Após ajuste para idade, o odds ratio para fibrilação atrial é 2,4 (2,1-2,9) em homens e $3,5(3,0-4,1)$ em mulheres. Após ajuste multivariado, passa a ser $1,5(1,2-$ $1,8)$ em homens e 1,9 (1,6-2,3) em mulheres. Portanto, portar a condição faz com que homens e mulheres se assemelhem quanto à mortalidade, reduzindo de 1,6 para 1,2 a razão entre $O R$ para homens e $O R$ para mulheres ${ }^{7}$. As mulheres tendem a ser mais velhas ao diagnóstico ${ }^{5,11}$, e, uma vez diagnosticadas, têm menor qualidade de vida ${ }^{11}$. As razões para isso ainda não foram esclarecidas.

A Iniciativa de Saúde da Mulher (Women's Health Initiative) realizou ensaios clínicos para avaliar o efeito de terapia de reposição hormonal durante a menopausa sobre fibrilação atrial e outras afecções cardiovasculares. Dados anteriores sugeriam que o aumento associado à idade no risco de eventos cardiovasculares em mulheres poderia ser eliminado com a terapia estrogênio-progestina ${ }^{12}$. Entretanto, os estudos do grupo demonstraram que não houve proteção contra doença arterial coronariana e houve aumento no risco de acidente vascular cerebral no grupo submetido à terapia. Houve aumento moderado na incidência de fibrilação atrial entre mulheres histerectomizadas que receberam terapia estrogênioprogestina, atribuído aos efeitos do conjugado estrogênio equino(CEE) em mulheres previamente histerectomizadas. Tal aumento diminuiu quando padronizado por incidência de doença arterial coronariana e insuficiência cardíaca, sugerindo que o efeito da terapia no desenvolvimento de fibrilação atrial pode ser indireto, devido ao aumento na incidência de doença cardiovascular ${ }^{11}$.

No passado, a doença cardíaca reumática (DCR) era a principal causa de fibrilacão atrial no mundo ${ }^{6}$. Estudos mostravam prevalência de fibrilação atrial de até $75 \%$ em pacientes com valvopatia secundária a doença cardíaca reumática ${ }^{13}$. Trata-se de uma das manifestações clínicas da febre reumática, uma complicação tardia, inflamatória, não supurativa, de base imunológica recidivante e que, frequentemente, atinge indivíduos de 5 a 15 anos de idade, de ambos os sexos, após infecções repetidas de faringoamigdalites pelo estreptococo beta-hemolítico do grupo A1. É a principal causa de doença cardiovascular adquirida na infância e na adolescência ${ }^{14}$. Atualmente, cerca de 30 milhões de pessoas são acometidas pela doença em todo o mundo ${ }^{15}$. Em países desenvolvidos, a prevalência da doença vem diminuindo em resposta às melhorias das condições socioeconômicas, culturais e do uso de antibióticos para infecções respiratórias. No Brasil, a prevalência da febre reumática é de 3 a $5 \%$ de crianças e adolescentes.

Contudo, estudos da segunda metade do século XX tem demonstrado uma transição na etiologia de fibrilação atrial $^{16}$. Um estudo com 1.212 pacientes entre 1940 e 1967 mostrou que no perfil etiológico da doença predominava doença cardíaca reumática no início do período e doença cardíaca isquêmica no fim do período ${ }^{19}$. Tal evidência pode explicar por que doença cardíaca reumática representa um fator etiológico importante no estudo de Framingham, mas não em estudos posteriores. A doença está praticamente extinta em países desenvolvidos ${ }^{16}$.

Um estudou observacional retrospectivo avaliou a prevalência de fibrilação atrial em 1.110 pacientes (média de idade \pm desvio padrão: $37,8 \pm 12,8$ anos) entre 1990 e 1994 com diagnóstico de doença cardíaca reumática, buscando estudar variáveis que contribuem para o 
desenvolvimento de fibrilação atrial. Desses, 433 pacientes tinham fibrilação atrial (FA), com idade 43.1 +- 14.4 anos. De 854 pacientes com estenose mitral, 403 (47\%) tinham FA. De 250 pacientes com estenose mitral isolada, 73 (29\%) tinham FA, e de 460 pacientes com estenose mitral e insuficiência mitral e/ou tricúspide, 270 (58\%) tinham FA. As valvopatias mitrais mostraram-se mais associadas a fibrilação atrial do que as valvopatias aórticas. Apenas 5 de $154(3 \%)$ dos pacientes com valvopatia aórtica tinham FA. As medidas de diâmetro de átrio esquerdo (DAE), realizadas por meio de ecocardiografia, mostraram que os maiores diâmetros foram encontrados em pacientes

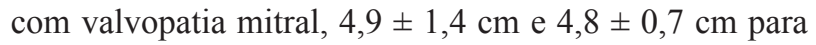
insuficiência e estenose mitral, respectivamente. Os diâmetros de átrio esquerdo foram significativamente menores em pacientes com valvopatia aórtica quando comparados aos pacientes com valvopatia mitral $(\mathrm{p}<0,0001)^{6}$.

Foram realizadas análises multivariadas incluindo covariantes comoidade, gênero, diâmetro deátrio esquerdo, gradiente diastólico mitral, gradiente aórtico, insuficiência aórtica, insuficiência mitral, insuficiência tricúspide e diâmetro diastólico final de ventrículo esquerdo. Dessas, idade e diâmetro de átrio esquerdo representaram fatores preditivos de fibrilação atrial, com risco relativo de 1,1 $(1,04-1,17)$ e $10,7(3,23-35,6)$, respectivamente 6 , sendo o diâmetro de átrio esquerdo o parâmetro mais importante na diferenciação de pacientes com e sem FA. O diâmetro de átrio esquerdo foi 5,7 $\pm 1,2$ e 4,0 $\pm 0,7 \mathrm{~cm}$ em pacientes com e sem FA, respectivamente $(\mathrm{p}<0,0001)$. Estudos anteriores ${ }^{18}$ mostram que a frequência de FA é de $3 \%$ em pacientes com DAE menor que $4,0 \mathrm{~cm}$ e aumenta para $54 \%$ em pacientes com DAE maior que 4,0 cm (Figura 5).

A fibrilação atrial observada em pacientes com valvopatia secundária a doença cardíaca valvar deve-se a mudanças hemodinâmicas que provocam sobrecarga de pressão e aumento de diâmetro no átrio esquerdo ${ }^{19}$. Além disso, na doença cardíaca reumática, a presença de fibrose favorece a formação de circuitos de reentrada que levam à fibrilação atrial.
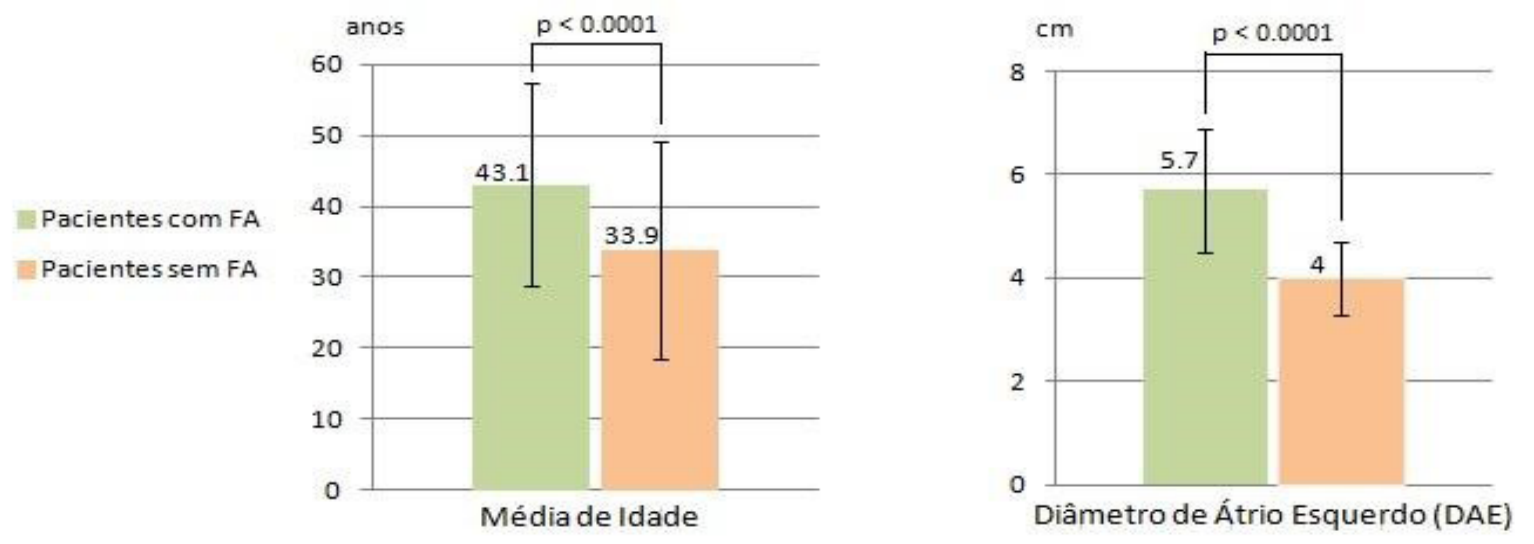

Figura 5 - Valores de média de idade e diâmetro de átrio esquerdo entre pacientes com fibrilação atrial e sem fibrilação atrial ${ }^{6}$

De acordo com outro estudo baseado na coorte de Framingham ${ }^{20}$, idade, diabetes melitus, hipertensão arterial sistêmica, insuficiência cardíaca congestiva e valvopatias representaram fatores de risco para fibrilação atrial em ambos os sexos. O OR para desenvolvimento de fibrilação atrial em vida foi 2,1 (1,8-2,5) em homens e 2,2 $(1,9-2,6)$ em mulheres. Insuficiência cardíaca congestiva teve $O R$ de $4,5(3,1-6,6)$ em homens e 5,9 $(4,2-8,4)$ em mulheres. Valvopatias tiveram $O R$ de $1,8(1,2-2,5) \mathrm{em}$ homens e 3,4 (2,5-4,5) em mulheres. Infarto agudo do miocárdio associou-se à fibrilação atrial em homens com $O R$ de 1,4 (1,0-2,0); enquanto em mulheres, o $O R$ foi de $1,2(0,8-1,8)$. Na análise do risco atribuível (RA), que leva em consideração o $O R$ e a prevalência do fator de risco e representa a porcentagem de casos de fibrilação atrial que poderiam ser evitados se determinado fator de risco fosse eliminado da população, insuficiência cardíaca congestiva teve RA de $10 \%$ para homens e $12 \%$ para mulheres; infarto do miocárdio, 5\% para homens e $1 \%$ para mulheres; valvopatias, $5 \%$ para homens e $18 \%$ para mulheres. Devido à alta incidência de hipertensão arterial sistêmica na população idosa, o RA foi 14\% para ambos os sexos. Hipertrofia ventricular esquerda e diabetes tiveram RA menor que $5 \%$.

O estudo escocês de Renfrew-Pasley ${ }^{21}$, que seguiu 15.406 pacientes de 1972 a 1976 e entre 1977 a 1979 , mostra que idade, histórico de acidente vascular cerebral, sintomas indicativos de ataque isquêmico transitório, evidência de infarto do miocárdio no eletrocardiograma, bloqueio de ramo esquerdo e cardiomegalia na radiografia de tórax foram fatores preditivos de fibrilação atrial.

Em estudo mais recente de Bruce et al. $^{22}$, que 
seguiu 5.201 de 1990 a 1993, idade, valvopatias, doença arterial coronariana, hipertensão arterial, altura, diabetes e tamanho do átrio esquerdo representaram fatores preditivos para fibrilação atrial. O uso de beta-bloqueadores, bebidas alcoólicas, volume expiratório forçado no primeiro minuto (VEF1) e níveis elevados de colesterol foram associados a risco diminuído de fibrilação atrial. Dentre pacientes com fibrilação atrial que receberam alta hospitalar, infarto do miocárdio (11\%), cirurgia de ponte aortocoronária (11,5\%), insuficiência cardíaca congestiva (19,6\%), valvopatia $(4,3 \%)$ e outras doenças cardiovasculares incluindo acidente vascular cerebral $(14,8 \%)$ foram as comorbidades mais prevalentes.

Dentre as outras causas de fibrilação atrial, destacam-se as cirurgias. A fibrilação atrial pós-operatória é uma complicação comum de cirurgias cardíacas, ocorrendo em 25 a $40 \%$ dos pacientes ${ }^{23}$. Muito prevalente, tal complicação vem associada a maiores períodos de internação e maiores riscos de acidente vascular cerebral no pós-operatório, elevando consideravelmente o custo total de cirurgia ${ }^{23}$. Ainda, se considerarmos que leitos para cuidados pós-operatórios são um fator limitante para a realização de cirurgias em países subdesenvolvidos, a prevenção de fibrilação atrial pós-operatória pode trazer benefícios adicionais em termos de saúde pública. Cinquenta e dois ensaios clínicos randomizados com anti-arrítmicos (beta-bloqueadores, sotalol e amiodarona) de 3 classes e marcapassos bicamerais mostraram que seu uso profilático reduz a incidência de fibrilação atrial pós-operatória e o período de internação pós-operatório (Figura 6) ${ }^{24}$. Contudo, não há evidências de que a taxa de acidente vascular cerebral sofra redução com o tratamento profilático, dado que a incidência de AVC após cirurgia cardíaca é baixa $(<3 \%)^{25}$.

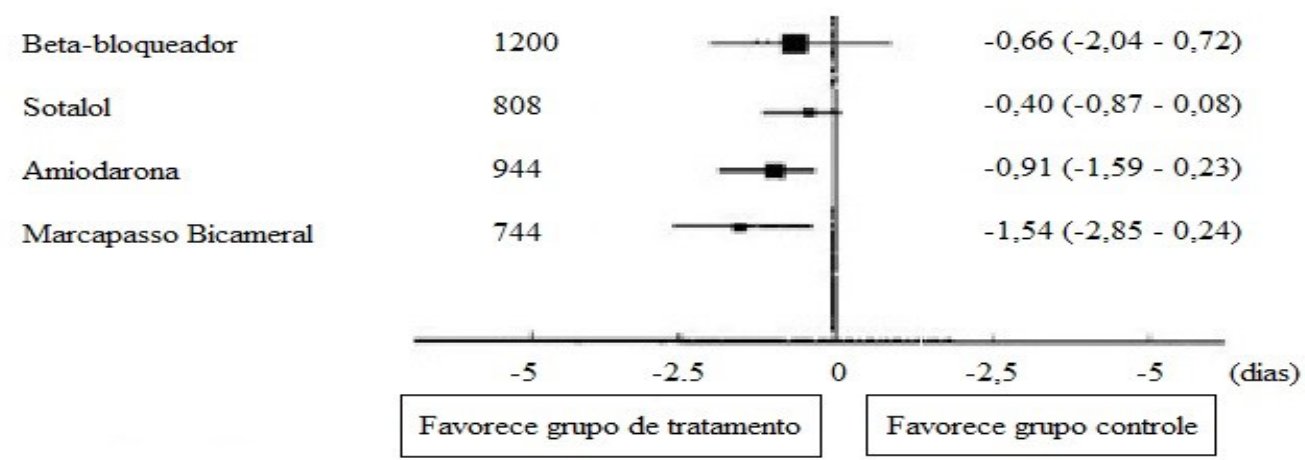

Figura 6 - Efeitos dos tratamentos profiláticos para fibrilação atrial no período de internação pós-operatória em dias ${ }^{24}$

\section{Perfil de morbimortalidade}

A morbidade e a mortalidade associadas à fibrilação atrial vêm tomando proporções maiores com o envelhecimento da população. Em 10 anos de seguimento de indivíduos entre 55 e 74 anos no estudo de Framingham, $61,5 \%$ dos homens com fibrilação atrial vieram à óbito, enquanto o mesmo ocorreu em 30,0\% dos homens sem fibrilação atrial; entre as mulheres, $57,6 \%$ das portadores de fibrilação atrial vieram à óbito, enquanto o mesmo ocorreu em 20,9\% das mulheres sem fibrilação atrial (Figura 7). A expectativa de vida a partir dessa faixa etária é menor para os portadores da doença. Entre os homens, é de 12,6 anos para os portadores e 18,1 anos para os não-portadores. Entre as mulheres, é de 12,1 anos para portadores e 21,3 para não-portadoras ${ }^{7}$. Nesse estudo, do total de 5.209 pacientes, os 621 pacientes que desenvolveram fibrilação atrial apresentaram mais fatores de risco cardiovasculares e condições/hábitos preexistentes, tais como hipertensão, hipertrofia ventricular esquerda, infarto do miocárdio, insu- ficiência cardíaca, valvopatia, tabagismo, acidente vascular cerebral e ataque isquêmico transitório ${ }^{7}$.

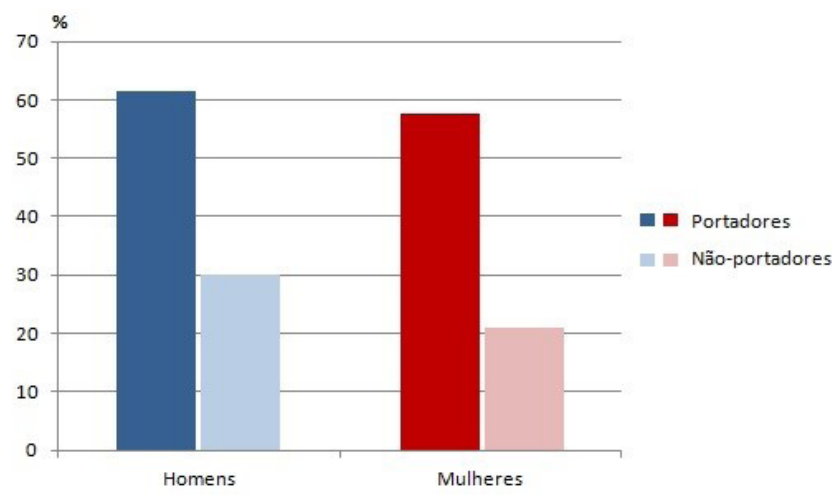

Figura 7 - Porcentagem de indivíduos que vieram à óbito em 10 anos de seguimento do estudo de Framingham ${ }^{7}$ 
Assim, para analisar a mortalidade atribuída à fibrilação atrial, realizou-se análise de regressão logística, cujos resultados sugerem que a maior parte da mortalidade é de fato devida à fibrilação atrial, e não um reflexo da carga de fatores de risco e condições preexistentes nos portadores da doença (Figura 8). Os resultados mostram que o $O R$ de fibrilação atrial ajustado para idade é 2,4 $(2,1-2,9)$ em homens e 3,5 (3,0-4,1) em mulheres. Após ajuste multivariado para idade e fatores de risco, o $O R$ de fibrilação atrial foi 1,5 (1,2 a 1,8) em homens e 1,9 (1,5 a 2,2) em mulheres, o que evidencia que fibrilação atrial continua associada à maior mortalidade ${ }^{7}$ Entretanto, a mortalidade associada à fibrilação atrial varia em relação ao gênero. A presença de fibrilação atrial faz com que homens e mulheres assemelhem-se quanto à mortalidade, conforme apresentado no Perfil Epidemiológico, reduzindo de 1,6 para 1,2 a razão entre $O R$ de homens e
$O R$ de mulheres ${ }^{7}$. A maior parte dos óbitos associados à fibrilação atrial foi precoce: aproximadamente $15 \%$ dos portadores vieram à óbito nos primeiros 30 dias após o diagnóstico. A análise dos indivíduos que permaneceram no estudo mostrou que fibrilação atrial também associa-se à maior mortalidade tardia em mulheres $(O R$ $1,5[1,2-1,8])$, mas não em homens $(O R \text { 1,1 [0,9-1,4]) })^{7}$. $\mathrm{O}$ estudo da mortalidade por causas de óbito no primeiro ano sugere que a distribuição é similar entre portadores e não-portadores de fibrilação atrial. Entretanto, quando cada causa é comparada separadamente, a mortalidade por causa associada à fibrilação atrial é maior no primeiro ano após o diagnóstico. Por fim, a análise dos pacientes sem quaisquer fatores de risco ou condições preexistentes mostrou que a fibrilação atrial está associada ao dobro da mortalidade, com $O R$ de 2,4 (1,8 a 3,3) em homens e 2,22 $(1,6$ e 3,1$)$ em mulheres ${ }^{7}$.

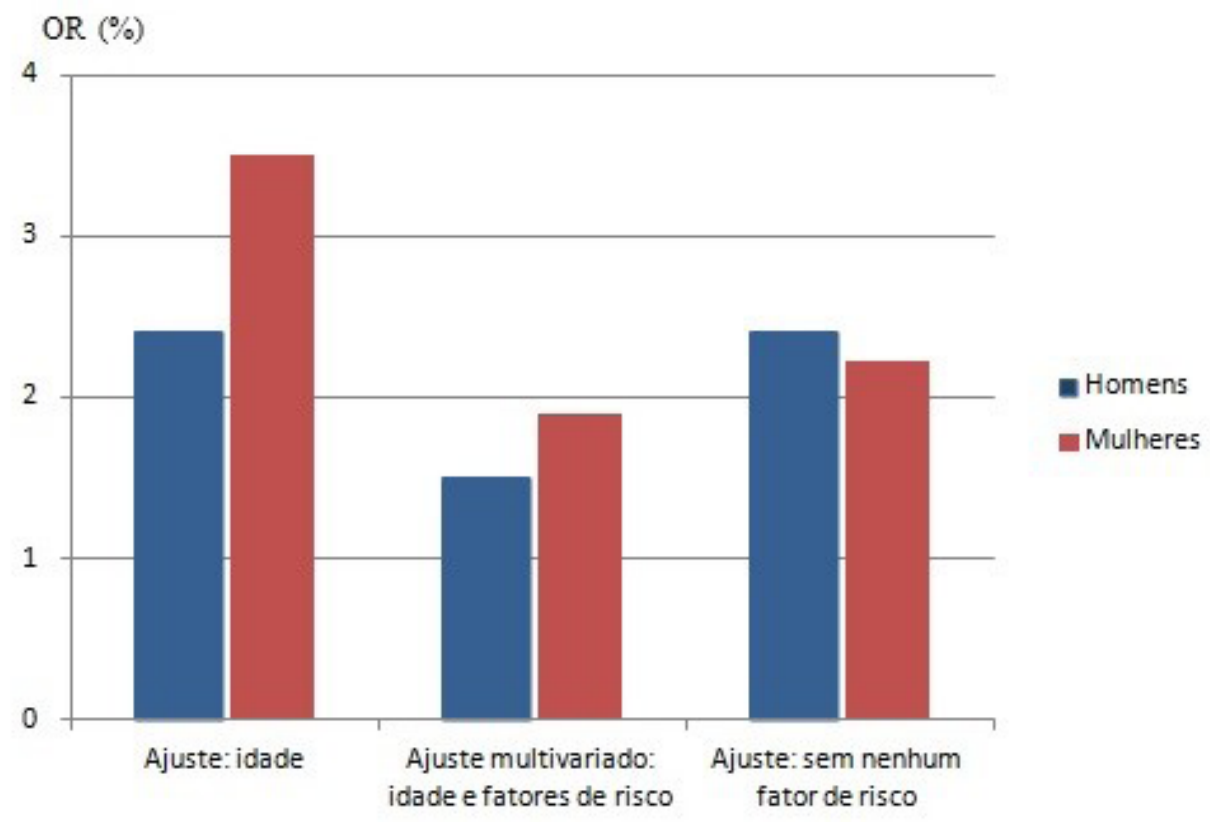

Figura 8 - Análise de regressão logística para avaliar a mortalidade atribuível à fibrilação atrial, com ajustes para idade; idade e fatores de risco; sem nenhum fator de risco ${ }^{7}$

Dentre as causas de óbito associadas à fibrilação atrial, destaca-se o acidente vascular cerebral, uma doença definida como déficit neurológico de causa cerebrovascular que persiste por mais de 24 horas ou que é interrompido por morte nas primeiras 24 horas. $\mathrm{O}$ acidente vascular cerebral associado à fibrilação atrial é do tipo isquêmico, resultante de embolia de origem cardíaca no átrio esquerdo ou na aurícula esquerda.

O risco de ocorrência de acidente vascular cerebral isquêmico é 5 vezes maior em portadores de fibrilação atrial. Para outras doenças cardiovasculares, o risco também aumenta: 2,4 vezes em presença de doença cardíaca coro- nariana, 3,4 vezes em presença de hipertensão, 4,3 vezes em presença de insuficiência cardíaca $(\mathrm{p}<0,001)^{25}$. Tais doenças não raro ocorrem concomitantemente à fibrilação atrial; nesse caso, a arritmia dobra e triplica o risco de AVC em homens e mulheres, respectivamente ${ }^{25}$. Com o avanço da idade, o risco de AVC atribuível à hipertensão, doença arterial coronariana e insuficiência cardíaca diminui progressivamente. O risco de $\mathrm{AVC}$ atribuível à fibrilação atrial, porém, aumenta de $1,5 \%$ no grupo etário de 50 a 59 anos para $23,5 \%$ no grupo etário de 80 a 89 anos, o que sugere que pacientes idosos portadores de fibrilação atrial são mais vulneráveis à incidência de $\mathrm{AVC}^{25}$ (Figura 9). 


\begin{tabular}{lcccc}
\hline & \multicolumn{3}{c}{ Grupo etário } \\
\cline { 2 - 5 } Doença cardiovascular & 50 a 59 anos & 60 a 69 anos & 70 a 79 anos & 80 a 89 anos \\
\hline Hipertensão & $48,8 \%$ & $53,2 \%$ & $48,6 \%$ & $33,4 \%$ \\
Doença cardiaca coronariana & $11,1 \%$ & $12,4 \%$ & $12,6 \%$ & $0,0 \%$ \\
Insuficiência cardiaca & $2,3 \%$ & $3,1 \%$ & $5,6 \%$ & $6,0 \%$ \\
Fibrilação atrial & $1,5 \%$ & $2,8 \%$ & $9,9 \%$ & $23,5 \%$ \\
\hline
\end{tabular}

Figura 9 - Tabela adaptada de Wolf $\mathrm{PA}^{25}$ mostra risco de acidente vascular cerebral atribuível a doenças cardiovasculares por grupo etário

Um estudo norte-americano realizado em Madison, no estado de Wisconsin, buscou avaliar se fibrilação atrial estava relacionada à maior gravidade dos acidentes vasculares cerebrais isquêmicos agudos (AVCIA), quando comparada a outras etiologias. Para isso, foram revisados prontuários de pacientes com AVCIA entre 1990 a 2001. De 1.061 casos, 216 tinham fibrilação atrial ${ }^{26}$.

O estudo mostra que a fibrilação atrial está associada a AVCIA severos e a pior prognóstico. Há um aumento na frequência de infarto total de circulação anterior de 14,4 para $26,3 \%$, com concomitante queda na frequência de infarto lacunar de 31,8 para $20,1 \%$, o que corresponde a um aumento na frequência de medidas clínicas de severidade de AVCIA, tais como comprometimento sensorial, déficit cortical e fraqueza do membro acometido, além de aumento na frequência de pacientes acamados, que é de $23,7 \%$ entre pacientes não portadores de fibrilação atrial e de $41,2 \%$ entre pacientes portadores ${ }^{26}$. Após ajuste para idade e outros fatores de risco para AVC, a fibrilação atrial permanece como fator de risco independente para AVCIA severo, tanto em termos de frequência de infarto total de circulação anterior $(O R$ 1,97 [1,55-2,39]) quanto em termos de frequência de pacientes acamados (OR 2,23 [1,87-2$, 59])^{26}$. A severidade do AVCIA e a incapacitância provocada pioram com grupos etários sucessivos na presença e na ausência de fibrilação atrial. Contudo, comparando-se um mesmo grupo etário, a severidade e a incapacitância são piores dentre os portadores de fibrilação atrial ${ }^{26}$.

Alguns estudos buscam medir o risco estratificado de AVC em pacientes com fibrilação atrial. Atualmente, utiliza-se o escore de $\mathrm{CHADS}_{2}$ para fibrilação atrial nãovalvar, que considera insuficiência cardíaca, hipertensão, idade acima de 75 anos e diabetes mellitus (cada um desses somando 1) e AVC (somando 2). Assim, pacientes com escore 0 apresentam baixo risco de AVC; pacientes com escore 1 a 2 apresentam risco moderado; e pacientes com escore maior que 2 apresentam alto risco. Embora simples e bem validado, o escore de $\mathrm{CHADS}_{2}$ apresenta limitações. Alguns estudos mostram que pacientes com escore 0 no $\mathrm{CHADS}_{2}(\operatorname{logo}$, de baixo risco e que não recebem anticoagulação) apresentam incidência anual de AVC de 1,4\% ${ }^{27}$.

Muitos estudos têm sido realizados na tentativa de criar um escore mais preciso, e para tal têm-se buscado considerar uma gama maior de fatores de risco, bem como interações entre eles. Uma abordagem recente de estratificação de risco divide os fatores de risco de "maiores" (são eles: AVC prévio ou acidente isquêmico transitório e idade acima de 75 anos) e "clinicamente relevantes menores" (insuficiência cardíaca, hipertensão arterial sistêmica, diabetes mellitus, gênero feminino, idade entre 65 e 75 anos e doença aterosclerótica). Tal escore é representado pelo acrônimo $\mathrm{CHA}_{2} \mathrm{DS}_{2}$-VASc, em que as letras correspondem a, respectivamente, insuficiência cardíaca congestiva, hipertensão arterial sistêmica, idade acima de 75 anos (em dobro), diabetes mellitus, AVC ou AIT (em dobro), doença vascular, idade entre 65 e 74 anos e gênero feminino ${ }^{27}$. De acordo com esse escore, pacientes com um fator de risco maior ou dois ou mais fatores de risco menores deveriam receber indicação de anticoagulação oral. Pacientes com apenas um fator de risco menor poderiam receber indicação de anticoagulação oral ou aspirina, mas preferencialmente anticoagulação oral. Pacientes sem fatores de risco (escore 0) poderiam receber indicação de aspirina ou não receber indicação alguma de trombolíticos, esta última conduta dado o baixo risco de AVC e a possibilidade de hemorragias $^{27}$. $\mathrm{O}$ escore $\mathrm{CHA}_{2} \mathrm{DS}_{2}$-VASc foi validado em um estudo inglês ${ }^{28}$ com 1.084 pacientes com fibrilação atrial não-valvar seguidos por um ano, em que houve associação entre maiores taxas de eventos tromboembólicos e escores mais altos, e pacientes com escore 0 não apresentaram nenhum evento tromboembólico. Entretanto, tal escore ainda necessita de validação em estudos prospectivos de populações diversas que contenham uma amostra mais representativa.

Ainda dentre as causas de óbito associadas à fibrilação atrial, destaca-se também a insuficiência cardíaca, "síndrome clínica complexa caracterizada por anormalidades de função ventricular esquerda e da regulação neuro-hormonal acompanhadas de intolerância ao esforço, retenção hídrica e redução da longevidade" 29 . Com o envelhecimento da população e o aumento na longevidade de pacientes cardiopatas com acesso a terapêuticas modernas, a prevalência de insuficiência cardíaca é cada vez maior, atingindo 5,1 milhões de norte-americanos em $2011^{30}$. Apesar de avanços 
no tratamento da doença, a mortalidade em pacientes com insuficiência cardíaca ainda é muito alta, tornando cruciais medidas preventivas que atuem em fatores de risco.

A fibrilação atrial é fator de risco para o desenvolvimento de insuficiência cardíaca, atuando por meio de mecanismos fisiopatológicos complexos que envolvem remodelamento atrial, miopatia induzida por taquicardia, alterações no sistema neuro-hormonal e alterações de parâmetros eletrofisiológicos e mecânicos. As duas doenças têm forte interação, com dados que mostram que $40 \%$ dos pacientes com ambas as doenças desenvolveram primeiro fibrilação atrial, seguida de insuficiência cardíaca ${ }^{31}$.

Um estudo norte-americano recente utilizou dados do estudo de Framingham para realizar análises multivariadas de 725 pacientes seguidos por 10 anos com fibrilação atrial $^{31}$. A média de idade dos pacientes foi 73,3 anos, e 45\% eram mulheres. Durante o seguimento, 161 pacientes desenvolveram insuficiência cardíaca; $45 \%$ eram mulheres ${ }^{31}$. A análise de incidência cumulativa em grupos etários mostrou maior incidência associada a pacientes mais velhos, com incidência total acumulada de $20 \%$ em indivíduos abaixo de 70 anos de idade e $41 \%$ em indivíduos acima de 70 anos de idade. Não houve diferenças associadas ao gênero. Modelos de riscos proporcionais de Cox ajustados para idade e sexo mostraram que idade (OR 1,05 [1,03-1,06]), índice de massa corporal (OR 1,06 [1,03-1,09]), peso (OR 1,02 [1,00$1,03])$, pressão sistólica elevada $(O R$ 1,01. [1,001-1,015]), diabetes mellitus (OR 2,15 [1,51-3,10]), sopros cardíacos (OR 1,77 [1,15-2,72]), hipertrofia ventricular esquerda ao ecocardiograma $(O R 2,11[1,38$ - [3,26]), infarto do miocárdio $(O R 2,26$ [1,59-3,21]) e doença cardiovascular $(O R$ $1,99[1,44-2,74])$ foram associados à maior incidência de insuficiência cardíaca. A relevância de tais fatores de risco foram similares em homens e mulheres ${ }^{31}$.

Enfim, a importância atribuída à fibrilação atrial em termos de saúde pública justifica-se, haja vista a magnitude da morbimortalidade associada à doença, com destaque para doenças cardiovasculares como o acidente vascular cerebral isquêmico e a insuficiência cardíaca, doenças letais e muito prevalentes cujos fatores de risco constituem alvo potencial de intervenção médica.

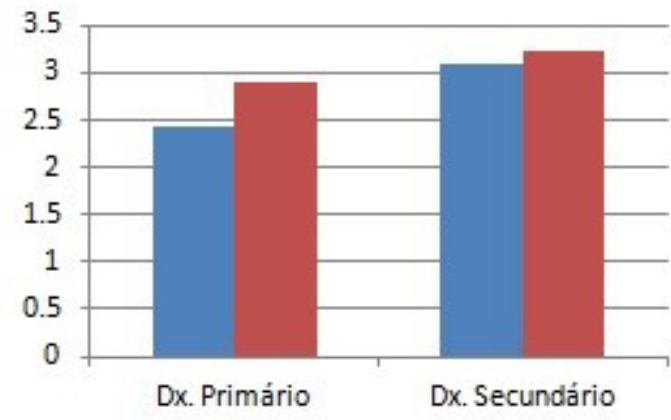

EUA

- Escócia

Figura 10 - Aumento no número absoluto de pacientes hospitalizados com diagnóstico primário ou secundário de fibrilação atrial entre as décadas de 1980 e 1990 nos EUA e na Escócia ${ }^{32,33}$

\section{Perfil de hospitalização}

A tendência de aumento na incidência de fibrilação atrial observada a partir da década de 1980, bem como o envelhecimento populacional e a incidência de outras afecções cardiovasculares, vem sendo acompanhada por aumento de hospitalizações de pacientes com diagnóstico de fibrilação atrial, o que se traduz em gastos de até 6 bilhões de dólares atribuídos exclusivamente à doença em uma população com cerca de 2,7 milhões portadores, como é o caso dos Estados Unidos, que calculam em 8.705 dólares o gasto adicional por paciente portador de fibrilação atrial em relação a um paciente de mesmo perfil não portador de fibrilação atrial. A previsão é de que, em 2050, haja entre 5,6 e 12 milhões de portadores da doença no país ${ }^{30}$.

Tendo em vista a incidência de fibrilação atrial, tornou-se de suma importância que se caracterizasse o quadro de hospitalizações atribuídas à doença, com ênfase em informações dos pacientes, dias de internação, prognóstico, mortalidade intra-hospitalar, entre outros. Dois estudos doravante vastamente comentados fornecem um esboço sobre o perfil de hospitalizações. O estudo americano de Wendy et al. ${ }^{33}$ utilizou-se do banco de dados norte-americano National Hospital Discharge Survey (NHDS) para obter dados de pacientes acima de 35 anos de idade com fibrilação atrial diagnosticada em um total de até sete diagnósticos, de acordo com o CID 9 (Classificação Internacional de Doenças, nona edição). O estudo escocês de Stewart et al. ${ }^{32}$ utilizou-se do banco de dados escocês Information and Statistics Division of the National Health Service para obter dados de pacientes com fibrilação atrial diagnosticada em um total de até seis diagnósticos, de acordo com o CID 9.

O número absoluto de pacientes hospitalizados com diagnóstico de fibrilação atrial aumentou significativamente nos dois estudos, comparando-se as décadas de 1980 e 1990 (Figura 10). No norte-americano, hospitalizações de pacientes com fibrilação atrial aumentaram 2,44 e 2,89 vezes como diagnóstico principal e diagnóstico secundário, respectivamente. No escocês, os aumentos foram de 3,08 e 3,22 , sendo fibrilação atrial o diagnóstico principal de $7 \%$ do total de hospitalizações por causas cardiovasculares e de $8 \%$ do total de dias de internação por causas cardiovasculares e o diagnóstico secundário de $20 \%$ do total de hospitalizações por causas cardiovasculares e de $41 \%$ do total de dias de internação por causas cardiovasculares. 
Nos dois estudos, a prevalência de fibrilação atrial é maior para faixas etárias maiores. No norte-americano, pacientes acima de 75 anos de idade representam mais de $50 \%$ dos pacientes hospitalizados com fibrilação atrial em 1999 (Figura 11). No escocês, essa taxa subiu de 33\% para $35 \%$ para homens e de $56 \%$ para $60 \%$ para mulheres de 1986 para 1996, e a média de idade de pacientes hospitalizados subiu um ano comparando-se os dois períodos. Em ambos, mulheres hospitalizadas tendem a ser mais velhas do que homens hospitalizados, em média 8 anos segundo o artigo escocês, em que $60 \%$ das mulheres hospitalizadas estão acima dos 75 anos, enquanto para os homens essa taxa é de 35\%. O número absoluto de mulheres hospitalizadas supera o de homens, mas quando padronizada por idade a prevalência de hospitalizações é maior em homens. O aumento no número de hospitalizações foi mais acentuado para homens ${ }^{32,33}$.
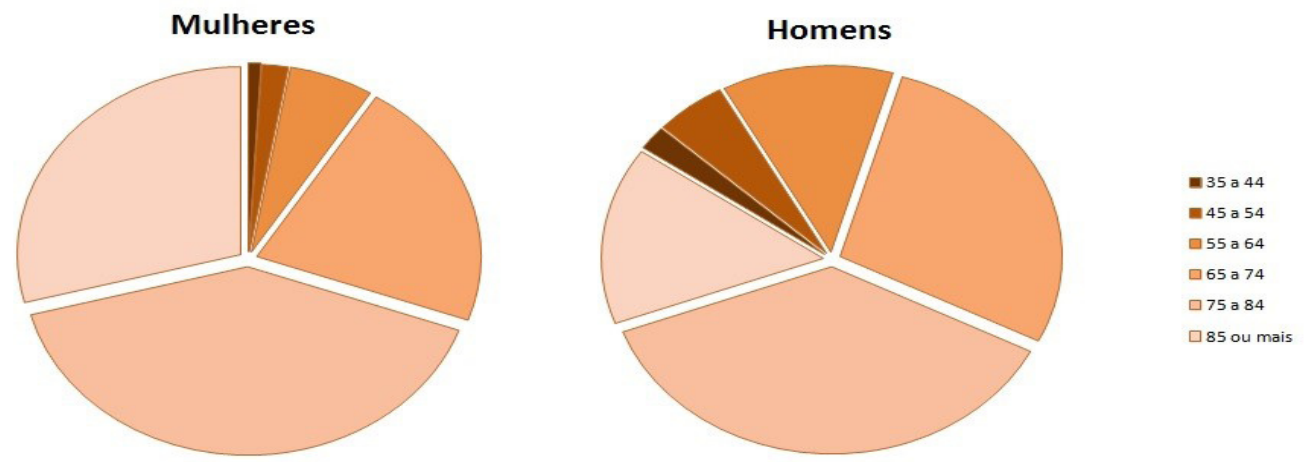

Figura 11 - Distribuição etária de pacientes hospitalizados com diagnóstico de fibrilação atrial na população norte-americana em $1999^{33}$

Nos casos em que fibrilação atrial é diagnóstico primário, os diagnósticos secundários mais prevalentes são hipertensão arterial sistêmica, infarto agudo do miocárdio e insuficiência cardíaca congestiva nas populações norteamericana e escocesa. Nos casos em que fibrilação atrial é diagnóstico secundário, os diagnósticos primários mais prevalentes são insuficiência cardíaca congestiva, aterosclerose e outras arritmias cardíacas na faixa etária de 35 a 64 anos de idade e insuficiência cardíaca congestiva, pneumonia e infarto agudo do miocárdio na faixa etária acima de 65 anos de idade na população norte-americana; e doença arterial coronariana, insuficiência cardíaca crônica e infarto agudo do miocárdio na população escocesa.

De 1986 a 1996, a população escocesa observou aumento de duas a três vezes tanto no número de hospitalizações por primeiro episódio de fibrilação atrial quanto no número de readmissões em um ano com o mesmo diagnóstico de fibrilação atrial. A letalidade sofreu queda no mesmo período, de $2,9 \%$ para $1,8 \%$ e de $13,0 \%$ para $7,8 \%$ em homens com fibrilação atrial como diagnóstico principal e secundário, respectivamente, e de $5,2 \%$ para $3,4 \%$ e $19,2 \%$ para $11,8 \%$ em mulheres com fibrilação atrial como diagnóstico principal e secundário, respectivamente, embora o número absoluto de mortes tenha dobrado em ambas as situações (Figura 12). Tal aumento deve-se à incidência crescente da doença, tendo sido maior entre mulheres, o que pode estar relacionado à idade avançada e à prevalência de acidente vascular cerebral, mais comum em mulheres ${ }^{32}$.
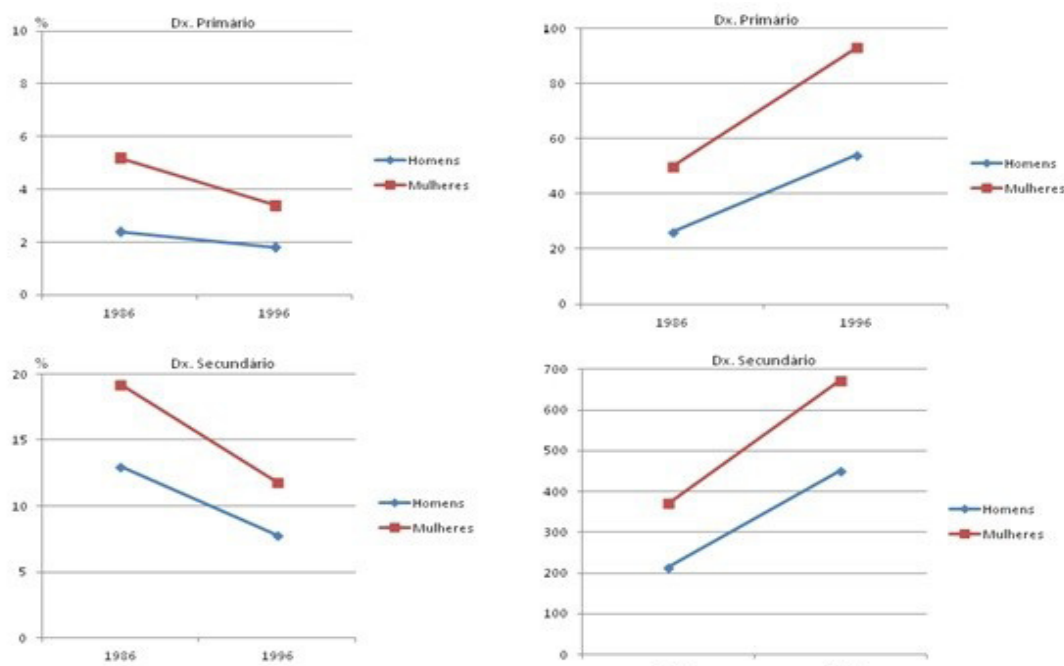

Figura 12 - À esquerda, queda porcentual na letalidade intra-hospitalar de fibrilação atrial como diagnóstico primário e secundário entre 1986 e 1996. À direita, aumento no número absoluto de pacientes com fibrilação atrial como diagnóstico primário e secundário que evoluíram a óbito entre 1986 e $1996^{32}$ 
De 1985 a 1999, a população norte-americana observou redução média de um a dois dias no tempo de internação por fibrilação atrial, com $72 \%$ das hospitalizações tendo duração de menos de uma semana. Essa redução vem acompanhada de queda no número de altas hospitalares, com aumento correspondente em encaminhamentos para cuidados a longo prazo e aumento discreto em encaminhamentos para cuidados a curto prazo, sem alteração na letalidade intra-hospitalar (Figura 13). Tal alteração no perfil de altas e encaminhamentos pode relacionar-se à prevalência de outras condições incapacitantes, destacada- mente a insuficiência cardíaca. O estudo norte-americano mostra ainda que não houve diferenças regionais nos perfis de hospitalização e que houve queda na participação do governo como responsável pelo pagamento, com aumento correspondente de seguradoras privadas. De 1986 a 1996, a população escocesa também observou redução média no tempo de internação, de 6 para 3 dias e 10 para 8 para homens com diagnóstico primário e secundário de fibrilação atrial, respectivamente, e de 8 para 5 dias e 13 para 11 para mulheres com diagnóstico primário e secundário de fibrilação atrial, respectivamente.
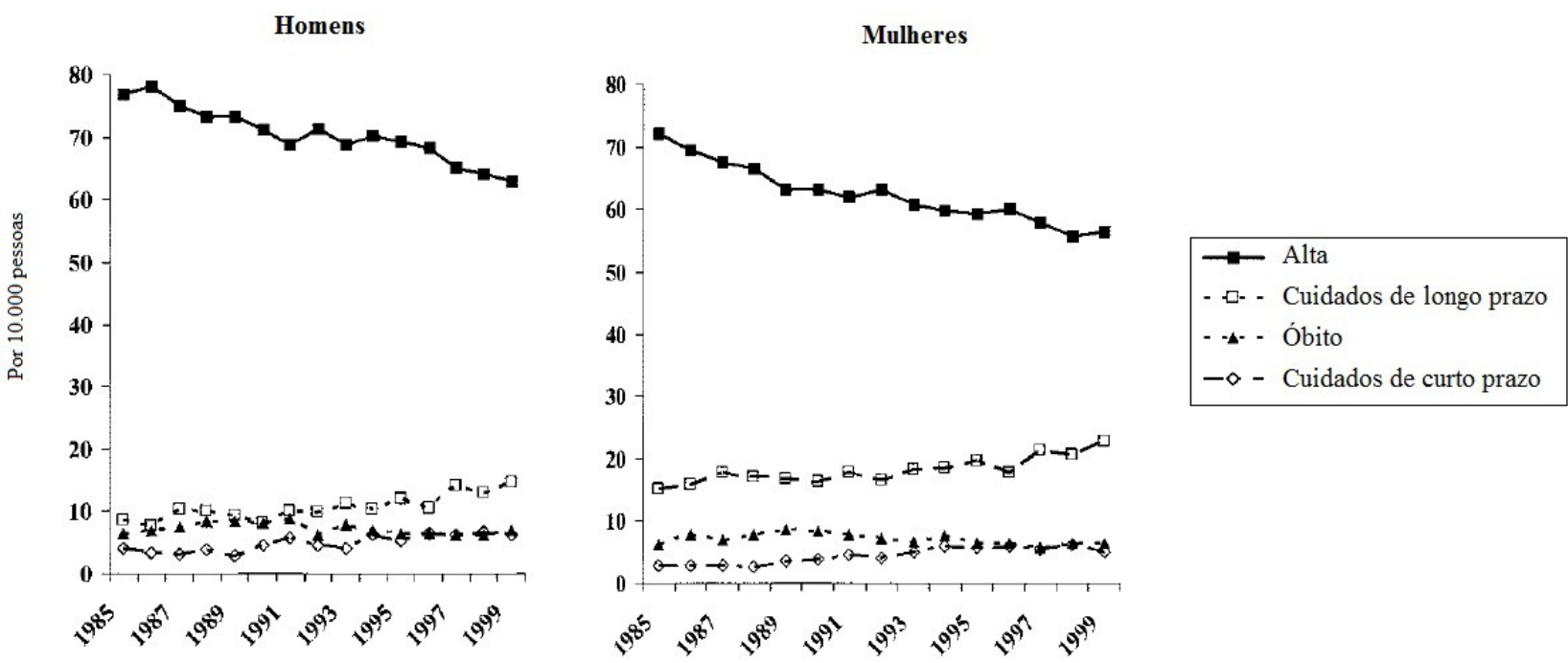

Figura 13 - Tabela adaptada de Wendy et al. ${ }^{33}$ mostra a distribuição proporcional de condições de pacientes hospitalizados com diagnóstico de fibrilação atrial por ano, de 1986 a 1996, na população escocesa

Em contraste com o aumento no número de hospitalizações por fibrilação atrial, o relatório do NHDS mostra que, nas estatísticas gerais, o número de hospitalizações caiu consideravelmente de 1985 a 1999, possivelmente por aumento de serviços ambulatoriais. Tal resultado acentua o aumento nas hospitalizações atribuídas à fibrilação atrial. Acredita-se que esse aumento tenha seguido a vanguarda científica, com a taxa de aumento de hospitalizações dobrando em 1990-1991, após a publicação de ensaios clínicos demonstrando a eficácia de terapia antitrombótica para prevenção de acidente vascular cerebral em pacientes com fibrilação atrial. O aumento de hospitalizações que, de 1986 a 1990, seguia a uma taxa de 6 a $10 \%$ ao ano, dobrou nos anos de 1990 e 1991, sugerindo mudanças no limiar para hospitalização e nos diagnósticos secundários associados à fibrilação atrial ${ }^{32}$.

Além disso, há nos trabalhos apresentados dados que fornecem informações valiosas se comparados a estudos anteriores. Diferente do estudo de Framingham, caracterizado por extenso inquérito populacional, os grupos aqui mencionados têm passagem hospitalar, o que pode explicar o maior perfil de morbidade cardiovascular dos mesmos, por exemplo a alta prevalência de insuficiência cardíaca, dado não encontrado no estudo de Framingham.

\section{CONCLUSÕES}

A fibrilação atrial está se tornando cada vez mais prevalente, especialmente entre indivíduos de idade avançada e indivíduos portadores de outras comorbidades cardiovasculares. Tal fato tem significado notório no atual panorama mundial, em que a expectativa de vida cresce na maioria dos países, bem como a incidência de doenças relacionadas à idade. Tem-se observado uma transição na etiologia da fibrilação atrial, com predomínio de doença cardíaca reumática na década de 1940 e atual predomínio de doença cardíaca isquêmica e doença hipertensiva. Homens têm maior prevalência de fibrilação atrial, embora estudos recentes mostrem que a doença faz com que a mortalidade em ambos os sexos se assemelhe, retirando a vantagem relativa das mulheres em sobrevida.

O aumento da prevalência de fibrilação atrial vem 
acompanhado do aumento da morbimortalidade atribuída à doença, com destaque para o risco de acidente vascular cerebral, cinco vezes maior nos portadores da arritmia. Apesar da redução no número de internações por causas gerais, as internações atribuídas à fibrilação atrial tiveram aumento significativo nas últimas décadas, bem como os encaminhamentos para serviços de cuidado a longo prazo,

\section{REFERÊNCIAS}

1. Lorga Filho A, Lorga AM, Lopes ANG, Paola AAV, Costa $\mathrm{AB}$, Péres AK, et al. Diretrizes de fibrilação atrial. Arq Bras Cardiol. 2003;81(supl 6):2-24. http://dx.doi.org/10.1590/ S0066-782X2003002000002.

2. ScherfD. Studies on auricular tachycardia caused by aconitine administration. Proc Exp Biol Med. 1947;64:233-9.

3. Haisseguere M, Jais P, Shah DC, Takahashi A, Hocini M, Quiniou G, Garrigue S, Le Mouroux A, Le Métayer P, Clémenty J. Spontaneous initiation of atrial fibrillation by ectopic beats originating in the pulmonary veins. NEngl J Med. 1998;339:659-66. doi: 10.1056/NEJM199809033391003.

4. Moe GK. Evidence for reentry as a mechanism of cardiac arrhytmias. Rev Physiol Biochem Pharmacol. 1975;72:5581.

5. Go AS, Hylek EM, Phillips KA, Chang Y, Henault LE, Selby JV, Singer DE. Prevalence of diagnosed atrial fibrillation in adults: national implications for rhythm management and stroke prevention: the AnTicoagulation and Risk Factors in Atrial Fibrillation (ATRIA) Study. JAMA. 2001;285(18):2370-5. doi:10.1001/jama.285.18.2370.

6. Diker E, Aydogdu S, Ozdemir M, Kural T, Polat K, Cehreli S, Erdogan A, Göksel S. Prevalence and predictors of atrial fibrillation in rheumatic valvular heart disease. Am J Cardiol. 1996;77(1):96-8. doi: 10.1016/S0002-9149(97)89145-X.

7. Benjamin EJ, Wolf PA, D'Agostino RB, Silbershatz H, Kannel WB, Levy D. Impact of atrial fibrillation on the risk of death: The Framingham Heart Study. Circulation. 1998;98:946-52. doi: 10.1161/01.CIR.98.10.946.

8. Kannel WB, Abbott RD, Savage DD, McNamara PM. Epidemiologic features of chronic atrial fibrillation. N Engl J Med. 1982;306(17):1018-22. doi: 10.1056/ NEJM198204293061703.

9. Ohsawa M, Okayama A, Okamura T, Itai K, Nakamura M, Tanno K, Kato K, Yaegashi Y, Onoda T, Sakata K, Ueshima H. Mortality risk attributable to atrial fibrillation in middleaged and elderly people in the japanese general population. Circulation. 2007;71(6):814-9. http://dx.doi.org/10.1253/ circj.71.814.

10. Wilke T, Groth A, Mueller S, Pfannkuche M, Verheyen F, Linder R, Maywald U, Bauersachs R, Breithardt G. Incidence and prevalence of atrial fibrillation: an analysis based on 8.3 million patients. Europace. 2013;15(4):486- como mostram estudos das décadas de 1980 e 1990.

Dessa forma, os aspectos epidemiológicos da fibrilação atrial levantados no nesta monografia sugerem a necessidade de abordagem da doença como questão de saúde pública, haja vista sua relevância como causa de redução de qualidade de vida, de morte e de sobrecarga dos serviços de saúde.

93. doi: 10.1093/europace/eus333.

11. Perez MV, Wang PJ, Larson JC, Virnig BA, Cochrane B, Curb JD, Klein L, Manson JE, Martin LW, Robinson J, Wassertheil-Smoller S, Stefanick ML. Effects of postmenopausal hormone therapy on incident atrial fibrillation: the Women's Health Initiative randomized controlled trials. Circ Arrhythm Electrophysiol. 2012;5(6):1108-16. doi: 10.1161/CIRCEP.112.972224.

12. Stampfer MJ, Colditz GA, Willett WC, Manson JE, Rosner B, Speizer FE, Hennekens CH. Postmenopausal estrogen therapy and cardiovascular disease. Ten-year follow-up from the nurses' health study. N Engl J Med. 1991;325(11):75662. doi: 10.1056/NEJM199109123251102.

13. Wipf JE, Lipsky BA. Atrial fibrillation: thromboembolic risk and indications for anticoagulation. Arch Intern Med. 1990;150(8):1598-603. doi:10.1001/ archinte.1990.00040031598006.

14. Borges F, Barbosa MLA, Borges RB, Pinheiro OC, Cardoso C, Bastos C, Aras R. Características Clínicas Demográficas em 99 Episódios de Febre Reumática no Acre, Amazônica Brasileira. Arq Bras Cardiol. 2005;84(2):111-4. http:// dx.doi.org/10.1590/S0066-782X2005000200004.

15. Miyatake K, lzumi S, Okamoto M, Kinoshita N, Asonuma $\mathrm{H}$, Nakagawa H, et al. Semiquantative grading of severity of mitral regurgitation by real time two dimensional Doppler flow imaging technique. J Am Col Cardiol. 1986;7(1):82-8. doi: 10.1016/S0735-1097(86)80263-7.

16. Frost L, Engholm G, Møller H, Husted S. Decrease in mortality in patients with a hospital diagnosis of atrial fibrillation in Denmark during the period 1980-1993. Eur Heart J. 1999;20(21):1592-9. Available from: http:// eurheartj.oxfordjournals.org/content/20/21/1592.long.

17. Godtfredsen J. Atrial fibrillation. Etiology, course and prognosis [Thesis]. Copenhagen: Munskgaard, 1975.

18. Hemy WL, Morganroth J, Pearlman AS, Clark CE, Redwood DR, Itscoitz SB, Epstein SE. Relation between echocardiographitally determined left atria1 size and atria1 fibrillation. Circulation. 1976;53(2):273-9. doi: 10.1161/01. CIR.53.2.273.

19. Kannel WB, Abbott RD, Savage DD, McNamara PM. Epidemiologic features of chronic atrial fibrillation: the Framingham Study. N Engl J Med. 1982;306(17):1018-22. 
doi: 10.1056/NEJM198204293061703.

20. Benjamin EJ, Levy D, Vaziri SM, D'Agostino RB, Belanger AJ, Wolf PA. Independent risk factors for atrial fibrillation in a population-based cohort: the Framingham Heart Study. JAMA. 1994;271(11):840-4. doi:10.1001/ jama.1994.03510350050036.

21. Stewart S, Hart CL, Hole DJ, McMurray JJV. Population prevalence, incidence, and predictors of atrial fibrillation in the Renfrew/Paisley study. Heart. 2001;86:516-21. doi: 10.1136/ heart.86.5.516.

22. Psaty BM, Manolio TA, Kuller LH, Kronmal RA, Cushman M, Fried LP, White R, Furberg CD, Rautaharju PM. Incidence of and risk factors for atrial fibrillation in older adults. Circulation. 1997;96(7):2455-61. doi: 10.1161/01. CIR.96.7.2455.

23. Crystal E, Connolly SJ, Sleik K, Ginger TJ, Yusuf $\mathrm{S}$. Interventions on prevention of postoperative atrial fibrillation in patients undergoing heart surgery: a metaanalysis. Circulation. 2002;106(1):75-80. doi: 10.1161/01. CIR.0000021113.44111.3E.

24. Ommen SR, Odell JA, Stanton MS. Atrial arrhythmias after cardiothoracic surgery. N Engl J Med. 1997;336(20):1429-34. doi: 10.1056/NEJM199705153362006.

25. Wolf PA, Abbott RD, Kannel WB. Atrial fibrillation as an independent risk factor for stroke: the Framingham Study. Stroke. 1991;22(8):983-8. doi: 10.1161/01.STR.22.8.983.

26. Dulli DA, Stanko H, Levine RL. Atrial fibrillation is associated with severe acute ischemic stroke. Neuroepidemiology. 2003;22(2):118-23. doi:10.1159/000068743.

27. Lip GY, Halperin JL. Improving stroke risk stratification in atrial fibrillation. Am J Med. 2010;123(6):484-8. doi: 10.1016/j.amjmed.2009.12.013.

28. Lip GYH, Nieuwlaat R, Pisters R, Lane DA, Crijns HJ. Refining clinical risk stratification for predicting stroke and thromboembolism in atrial fibrillation using a novel risk factor based approach: The EuroHeart Survey on Atrial Fibrillation. Chest. 2010;137(2):263-72. doi: 10.1378/chest.09-1584.

29. Courneya C-A, Parker MJ, Schwartzstein RM. Cardiovascular physiology: a clinical approach (integrated physiology). Philadelphia: Lippincott Williams \& Wilkins; 2010.

30. Go AS, Mozaffarian D, Roger VL, Benjamin EJ, Berry JD, Borden WB, et al. Heart disease and stroke statistics--2013 update: a report from the American Heart Association. Circulation. 2013;127:e6-e245. doi: 10.1161/ CIR.0b013e31828124ad.

31. Schnabel RB, Rienstra M, Sullivan LM, Sun JX, Moser CB, Levy D, Pencina MJ, Fontes JD, Magnani JW, McManus DD, Lubitz SA, Tadros TM, Wang TJ, Ellinor PT, Vasan RS, Benjamin EJ. Risk assessment for incident heart failure in individuals with atrial fibrillation. Eur J Heart Fail. 2013;15(8):843-9. doi: 10.1093/eurjhf/hft041.

32. Stewart S, MacIntyre K, MacLeod MMC, Bailey AEM, Capewell S, McMurray JJV. Trends in hospital activity, morbidity and case fatality related to atrial fibrillation in Scotland, 1986-1996. Eur Heart J. 2001;22(8):693-701. doi: 10.1053/euhj.2000.2511

33. Wattigney WA, Mensah GA, Croft JB. Increasing trends in hospitalization for atrial fibrillation in the United States, 1985 through 1999: implications for primary prevention. Circulation. 2003;108(6):711-6. doi: 10.1161/01. CIR.0000083722.42033.0A 